\title{
Podolsky's Electrodynamics Via First Principles
}

\section{A Eletrodinâmica de Podolsky Via Princípios Primeiros}

Article Info:

Article history: Received 2021-11-11 / Accepted 2022-01-30/ Available online 2022-02-25

doi: $10.18540 /$ jcecvl8iss2pp13818-01e

Jorge Henrique de Oliveira Sales

ORCID: https://orcid.org/0000-0003-1992-3748

Universidade Estadual de Santa Cruz, Brasil

E-mail: jhosales@uesc.br

\begin{abstract}
A theory of electromagnetism with higher order derivatives, which attained by generalizing the laws of electrostatics, laws that follow from the generalized Coulomb's law and the superposition principle, so that they are consistent with special relativity.
\end{abstract}

Keywords: higher-order electromagnetism, Coulomb's generalized law, principle of superposition, special relativity.

\section{Resumo}

Obtém-se uma teoria de eletromagnetismo com derivadas de ordem mais alta generalizando-se as leis da eletrostática, leis estas que seguem da lei de Coulomb generalizada e do princípio de superposição, de modo que elas sejam consistentes com a relatividade especial.

Palavras-chave: eletromagnetismo com derivadas de ordem mais alta, lei de Coulomb generalizada, princípio de superposição, relatividade especial.

\section{Introduction}

The interest in theories with higher order derivatives has occupied the attention of physicists since the adoption of differential equations as a mechanism for describing physical systems (SALES; GIROTTO, 2021). It is noteworthy that Lagrangeans with higher order derivatives, which have already been discussed in Courant-Hilbert's, (COURANT, 1962).

Despite the interest in using these theories and it never been forgotten over time, it was considered purely academic (SALES,1995). This is because the most important systems in field theories are describe or Lagrangians can transform that by adding a total derivative term to the corresponding action.

With the advent of supersymmetry and string theory, the higher order theories have lost their academic stamp and the interest in them has increased. This is easy to understand, since both in supersymmetry, in its formulation in terms of super fields (BARCELOS, 1989, 1991a, 1991b), and in important examples in string theory (POLYAKOV, 1986), higher order derivatives occurred in abundance.

Also, utilize higher order derivatives as a regularization method in field theory (SLAVNOV, 1978). As far as we know, one of the first successful attempts at using terms containing higher order derivatives as a regularizing mechanism is due to Podolsky (PODOLSKY, 1942, 1944, 1945, 1948), who generalized Maxwell's electromagnetism in order to get rid of the infinities that occur in it.

In this paper, we will focus on Podolsky electrodynamics. We obtain the Podolsky equations by generalizing the laws of electrostatics (BARTLETT, 1977, 1979) that follow from the 
generalized Coulomb's law and the superposition principle, in such a way that they are consistent with special relativity.

\section{Podolsky's Propagator}

The generalized electrodynamics proposed by Podolsky is based on the Langrangean

$$
\mathcal{L}_{0}=-\frac{1}{4} \mathrm{~F}_{\mu \mathrm{v}} \mathrm{F}^{\mu \mathrm{v}}+\frac{\mathrm{a}^{2}}{2} \partial_{\mathrm{v}} \mathrm{F}^{\mu \mathrm{v}} \partial^{\mathrm{i}} \mathrm{F}_{\mu \mathrm{i}}
$$

where $F_{\mu v}=\partial_{v} A_{\mu}-\partial_{\mu} A_{v}$ and $a$ is a constant with length dimension. This Lagrangian generates a linear field theory, with gauge symmetry of the type $U(1)$, which reduces to Maxwell's theory when $a=0$. This is evidently a higher-order theory since the equations of motion derived from (1) contain quartic derivatives of the vector potential. Like Maxwell's theory, Podolsky's theory also has definite positive energy in the electrostatic case, which, however, is finite for a point charge. This last result clearly shows that the force between two-point charges is no longer Coulombian, a point that deserves to be analysed more closely. Let us then determine the law that describes the interaction between two point charges in the context of this electrodynamics with higher order derivatives.

As is well known, the exchange of a photon between elastically scattered particles naturally leads to the concept of force or, equivalently, to that of electrostatic potential. To determine the force law associated with Podolsky's theory we will analyse, in lower order, the process $s+s \rightarrow$ $s+s$, where s represents a boson of zero spin and charge $Q$. The corresponding Feynman diagram shown in Fig. 1.

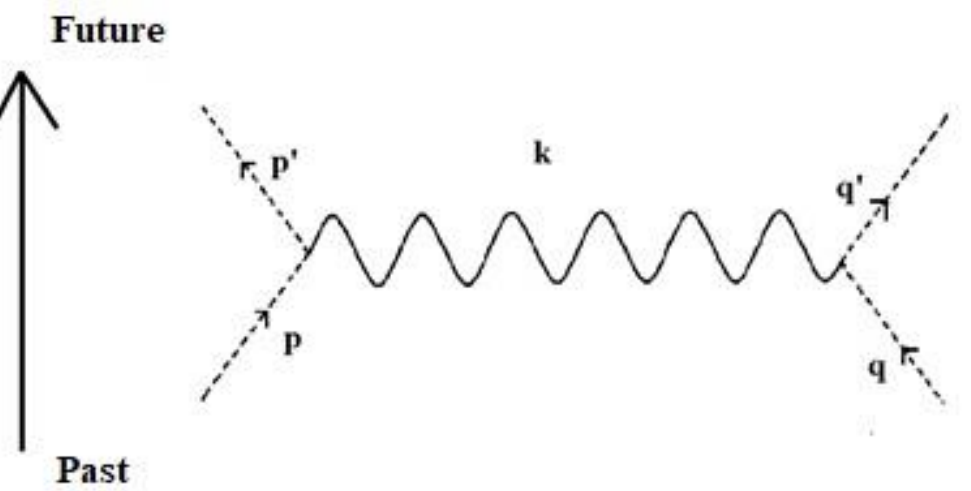

Figure 1. Contribution, in lowest order, to the process $s+s \rightarrow s+s$.

Source: SALES, 1995.

In the Lorentz gauge, the Lagrangean below describes Podolsky electrodynamics scalar:

$\mathcal{L}=-\frac{1}{4} F_{\mu v} F^{\mu v}+\frac{a^{2}}{2} \partial_{v} F^{\mu v} \partial^{\lambda} F_{\mu \lambda}-\frac{1}{2}\left(\partial_{\mu} A^{\mu}\right)^{2}+\left(D_{\mu} \phi\right)^{*} D^{\mu} \phi-m^{2} \phi^{*} \phi$

where $D_{\mu} \phi=\partial_{\mu} \phi+i Q A_{\mu}$.

At lower order, the interaction Lagrangian takes the form 


$$
\mathcal{L}_{\mathrm{I}}=i Q A^{\mu}\left(\phi \partial_{\mu} \phi^{*}-\phi^{*} \partial_{\mu} \phi\right)
$$

Can also, equivalent to Feynman rule be found in Fig. 2. Next, we will determine the propagator, in the Lorentz gauge, associated with Podolsky's generalized electrodynamics.

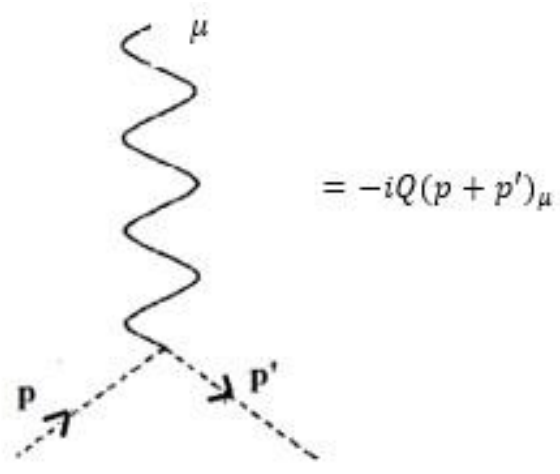

Figure 2. Elementary vertex for Podolsky scalar electrodynamics.

Source: SALES, 1995.

The free Lagrangian quadratic part of Podolsky, also can be written in the Lorentz gauge as

$\mathcal{L}=\frac{1}{2} A_{\mu}(x) P^{\mu v}(x) A_{v(x)}$

where

$$
P^{\mu v}(x)=n^{\mu v} \square+a^{2}\left(n^{\mu v} \square \square-\partial^{\mu} \partial^{v} \square\right)
$$

The (5) It follows that

$$
P^{\mu v}(k)=n^{\mu v}\left(a^{2} k^{4}-k^{2}\right)-k^{2} a^{2} k^{\mu} k^{v}
$$

Inverting (6), we obtain

$$
P_{\mu v}(k)=\frac{1}{a^{2} k^{4}-k^{2}}\left[n_{\mu v}-a^{2} k_{\mu} k_{v}\right]
$$

We define $M^{2}=\frac{1}{a^{2}}$ the expression (7) even as can be written in the form of propagator $D_{\mu \nu}(k)=i P_{\mu v}(k)$ or

$D_{\mu \nu}(k)=\frac{i M^{2}}{k^{2}\left(k^{2}-M^{2}+i \varepsilon\right)}\left\lceil n_{\mu v}-\frac{k_{\mu} k_{v}}{M^{2}}\right\rceil$

This propagator shows good ultraviolet behaviour $\left(\approx k^{-1}\right)$ thanks to the presence of a nontachyonic ghost.

\section{Podolsky's Electrostatics}

The Feynman invariant amplitude for the process shown in Fig. 1 even as can be written as 
$\mathcal{A}=i . V^{\mu}(1) \cdot V_{\mu}(2) \cdot \frac{M^{2}}{k^{2}\left(k^{2}-M^{2}\right)}$,

where

$$
\begin{aligned}
& V^{\mu}(1) \equiv-i Q\left(p+p^{\prime}\right)^{\mu} \\
& V^{\mu}(2) \equiv-i Q\left(q+q^{\prime}\right)^{\mu}
\end{aligned}
$$

Remembering that the conservation of the four-momentum $k=p-p^{\prime}=q-q^{\prime}$, (9) became

$\mathcal{A}=\frac{-i \cdot M^{2} Q^{2}}{k^{2}\left(k^{2}-M^{2}\right)}(2 p-k)(2 q+k)$

In the non-relativistic limit (10) it takes the form

$$
\mathcal{A}_{v \ll c}=\frac{-4 i M^{2} Q^{2} m^{2}}{\vec{k}^{-2}\left(\vec{k}^{-2}+M^{2}\right)}
$$

In possession of the expression for the non-relativistic limit of the Feynman amplitude invariant, we can calculate the electrostatic potential.

Electrostatic potential energy, in the Gaussian system, is expressed as follows:

$U(r)=\frac{1}{4 m^{2}} \cdot \frac{1}{(2 \pi)^{3}} \cdot \int d^{3} k \cdot F_{v \ll c}(k) \cdot \exp (i k \cdot r)$,

where

$$
F_{v \ll c}(k)=i \mathcal{A}_{v \ll c}(\mathrm{k})
$$

Taking (11) into (12) we arrive at the following expression for potential energy

$$
U(r)=\frac{Q^{2}}{4 \pi} \frac{1-e^{-r M}}{r}
$$

The electrostatic potential then takes the form

$$
V(r)=\frac{Q}{4 \pi} \frac{1-e^{-r M}}{r}
$$

This potential is finite in all space and cancels itself out at infinity

$$
\lim _{r \rightarrow \infty} V(r)=0
$$

Quantum electrodynamics (GOMES, 1990) shows that the long range of the Coulomb interaction is the result of the zero rest mass of the quanta exchanged in the interaction and that this range would not be long if the mass of the quanta were non-zero. Yukawa adapted the theory to the case of a system of nuclei, interacting through a short-range force, assuming that the "quanta" of the "field" that describes the interaction have non-zero mass. These "quanta" are called mesons and the "field" is called the mesonic field. The constant $M$ of Eq. 14 is identified as the mass of the mesons. We can estimate the mass as being around $m_{\pi} \sim 200 m$, where $m$ is the rest mass of the electron (FEYNMAN, 1965). 


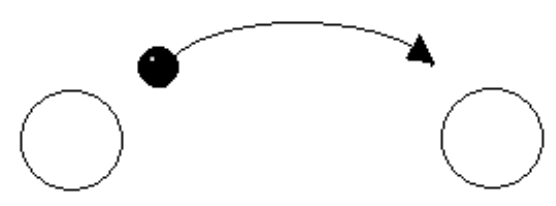

Figure 3. Schematic representation meson exchange process.

By the Langragian (2) it is observed that the constant $a$ is the distance. Thus, the value $M$ (mass) can be estimated as $M=m_{\pi} \sim h / a c$ (GOMES, 1990). Using the potential (14) for values $a=1,2,3$ and $Q=1$, result shown in figure 4 :

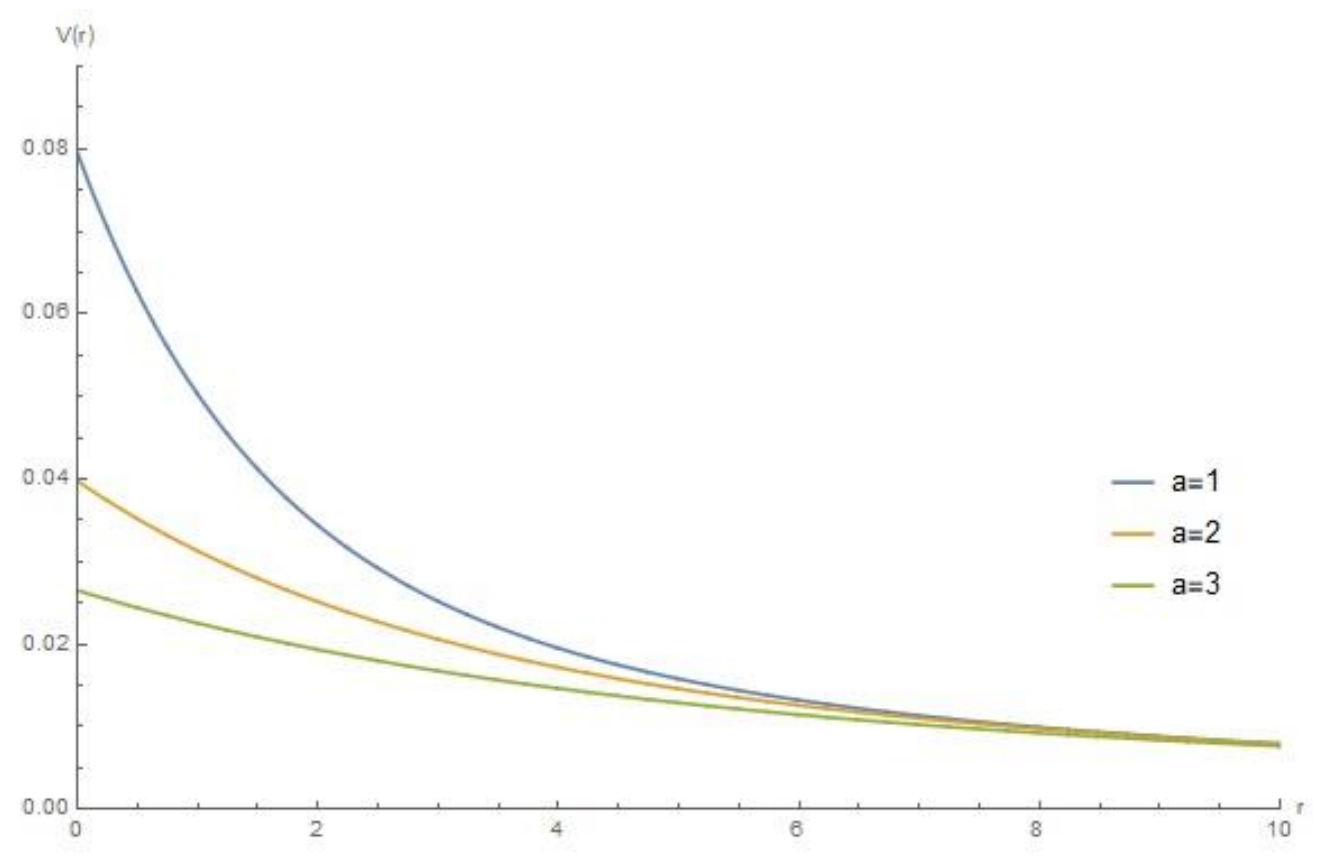

Figure 4. Potential varying with range $r$.

In figure 4 we do not consider the units of physical quantities. It is observed that smaller values for the distance $a$ imply in the increase of the potential $V(r)$. Therefore, meson exchange involves higher values for the potential. For much larger values of $a$ there is a lower limit to the potential for the three values of $a$. 


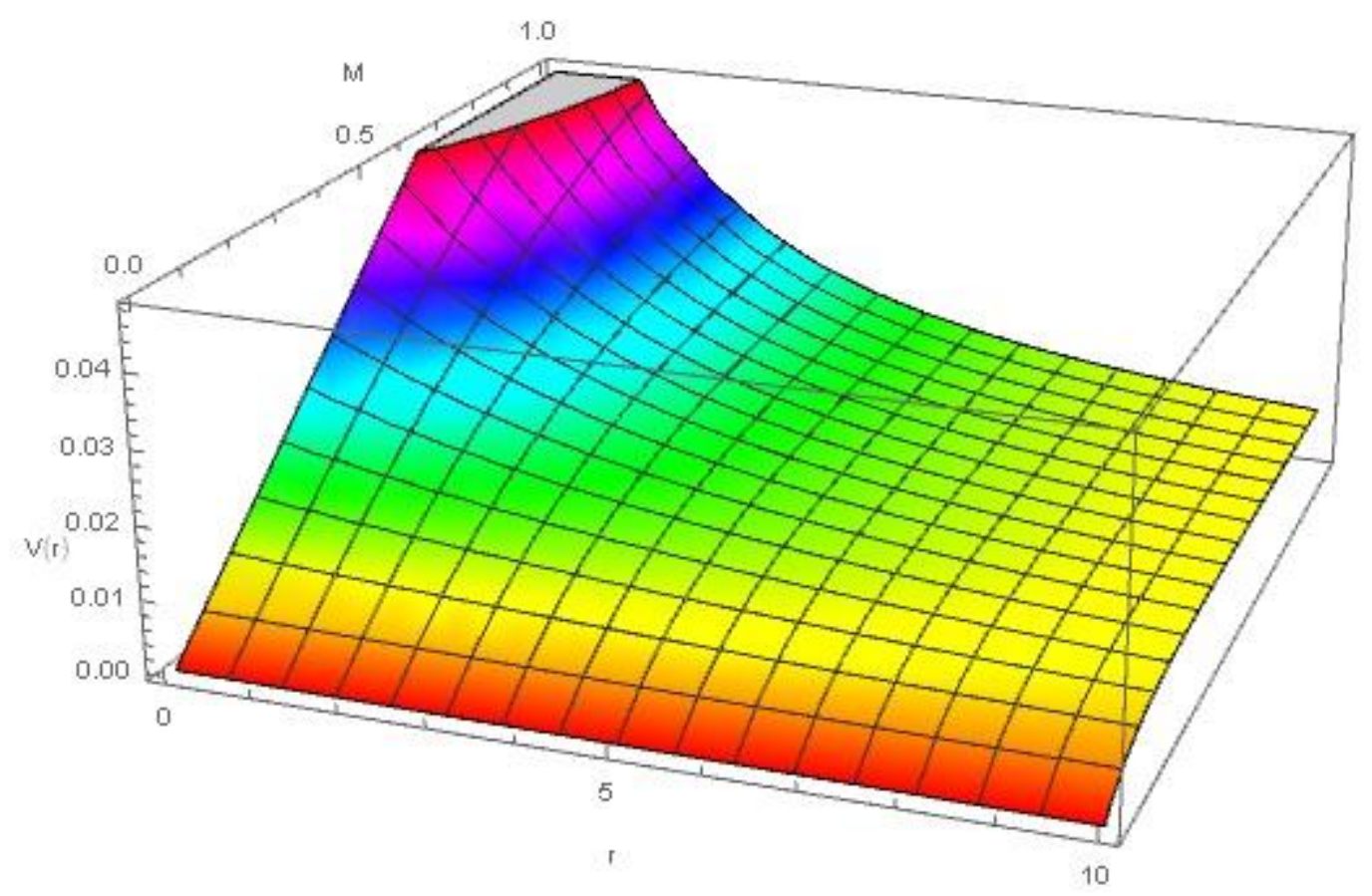

Figure 5. Potential varying with range $r$ and mass $M$.

In figure 5 we see that the variation of distance and mass results in a stronger potential. This suggests that the Podolsky potential is more general than the Maxwell electromagnetism potential. Therefore, for $r=0$ the potential is finite any $M$.

From (14) it immediately follows that the force law corresponding to the interaction between two points charges $Q$ and $Q^{\prime}$ it shows up:

Resulting in

$$
F(r)=-\frac{d V(r)}{d r}
$$

$\vec{F}(r)=\frac{Q Q^{\prime}}{4 \pi}\left(\frac{1-e^{-r M}}{r^{2}}-\frac{M e^{-r M}}{r}\right) \frac{\vec{r}}{r}$

From generalized Coulomb's law, Eq. (15), we get the electric field $E(r)$ at point $r$ due to a point charge $Q$ located by: $r=0$

$E(r)=\frac{Q}{4 \pi}\left[\frac{1-e^{-r M}}{r^{2}}-\frac{M e^{-r M}}{r}\right] \frac{\vec{r}}{r}$

It is interesting to note that the flux of this field through a spherical surface of radius $R$ centred on the charge $Q$ it shows up:

$$
\oint \vec{E} \cdot d \vec{S}= \begin{cases}0, & R M \ll 1 \\ Q, & R M \gg 1\end{cases}
$$

From generalized Coulomb's law and the superposition principle we get the electric field $\vec{E}(\vec{r})$ due to electrostatic charge density $\rho(\vec{r})$ (vide Fig. 6):

$$
\vec{E}(\vec{r})=\int d^{3} r^{\prime} \frac{\rho\left(\overrightarrow{r^{\prime}}\right)}{4 \pi}\left[\frac{1-e^{-R M}}{R^{2}}-\frac{M e^{-R M}}{R}\right] \frac{\vec{R}}{R},
$$


where $\vec{R} \equiv \vec{r}-\overrightarrow{r^{\prime}}$.

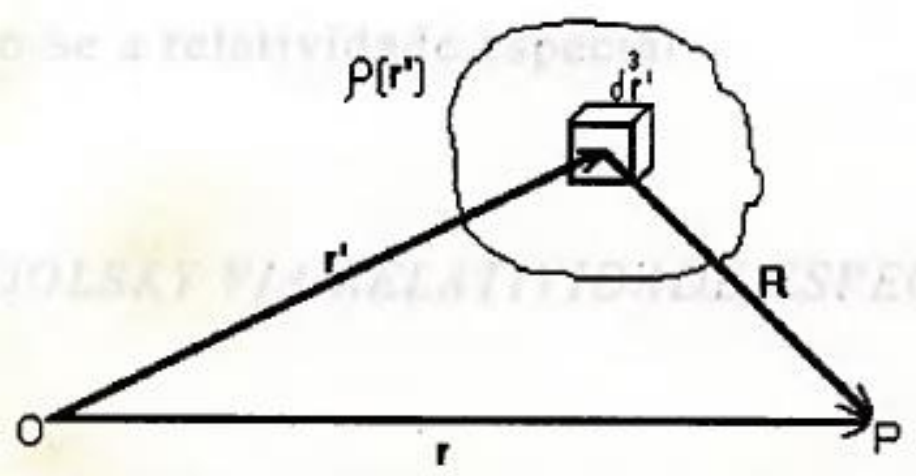

Figure 6. Geometry for determining the electrostatic field at $P$.

Source: Sales, 1995.

Using (17) and a little elementary algebra, we can write:

$\nabla \cdot \vec{E}(\vec{r})=\int d^{3} \vec{r}^{\prime} \rho\left(\vec{r}^{\prime}\right) \frac{M^{2}}{4 \pi} \frac{e^{-R M}}{R}$

From (18) followed

$\left(1-a^{2} \nabla^{2}\right) \nabla \cdot \vec{E}(\vec{r})=\rho(\vec{r})$

Which is the generalization of Maxwell's Gauss law of electrostatics. In obtaining (19) we use the result

$$
\nabla_{\vec{r}}^{2} \frac{e^{-R M}}{R}=\frac{M^{2}}{R} e^{-R M}-4 \pi \delta^{3}(\vec{R})
$$

Taking the rotation of (19), we obtain

$\nabla \times \vec{E}(\vec{r})=0$

What it shows? That just as in Maxwell's theory, the electrostatic field is also conservative. Equations (19) and (20) are the fundamental laws of Podolsky's electrostatics and will be generalized later using special relativity.

\section{Podolsky via Especial Relativity}

Since electric charge is supposed to be a globally conserved scalar (FEYNMAN, 1965), it follows that the charge density transforms as the zero component of a 4-vector. This 4-vector is the current density $j^{\mu}\left(j^{0}, \vec{\jmath}\right)$ where $j^{0}=\rho$.

The global conservation of electric charge implies its local conservation (KOBE, 1986). We lead to the continuity equation for the electric charge

$\partial_{\mu} j^{\mu}=0$

which are valid in all Lorentz referential. 
We can now generalize to the left (19), requiring it to be shape-variant when subjected to a Lorentz transformation. The left side (19), can be rewritten in terms of $j^{0}$ as

$$
\left(1-\mu^{2} \nabla^{2}\right) \partial_{i} E^{i}=j^{0}
$$

where $i=1,2,3$. The right side of (22) is the zero component of a 4-vector, which implies that the right-hand side must also transform as the zero component of a 4-vector. If $F^{\mu v}$ is a second order tensor, so $\partial_{v} F^{\mu v}$ is transformed as the zero component of a 4-vector. It is not difficult to show that the $E^{i}$ of the electric field can be expressed with the following components of a second-order tensor:

$F^{01}=E^{1}, F^{02}=E^{2}, F^{03}=E^{3}$

By induction from equation (22) with help (23), it follows that:

$\left(1+a^{2} \eta^{i j} \partial_{i} \partial_{j}\right) \partial_{v} F^{0 v}=j^{0}$

where $F^{00}$ will be specified later and $\eta^{\mu v}$ is the metric versus variant tensor of special relativity that in our convention has the following components (BJORKEN; DRELL, 1964):

$\eta^{00}=-\eta^{11}=-\eta^{22}=-\eta^{33}=1$.

Now both sides of (24) become the zero component of a 4-vector. In order for the laws of physics to be, form invariant with respect to Lorentz transformations (24), it will be led to generalization as

$\left[1+a^{2} \square\right] \partial_{v} F^{\mu v}=j^{\mu}$,

where $\square=\eta^{\mu v} \partial_{\mu} \partial_{v}=\partial_{\mu} \partial^{\mu}$

Using (25), we get:

$\left[1+a^{2} \square\right] \partial_{\mu} \partial_{v} F^{\mu v}=0$

If $F^{\mu v}$ is antisymmetric, so (26) is identically null. Evidently (26) does not imply that $F^{\mu v}$ be antisymmetrical. This, however, is the simplest solution for the equation (25) which we will adopt. Let's then choose the three remaining components from $F^{\mu v}$ such as

$F^{i 2}=B^{3}, F^{i 3}=-B^{2}, F^{23}=B^{i}$.

and $\vec{B}=\left(B^{1}, B^{2}, B^{3}\right)$ Commonly interpreted physically as the magnetic induction field.

The left (25), relativistic version of the (19), it is one of the equations of the pair of equations proposed by Podolsky. Generalizing (20) obtain the missing equation. Using the Levi-Civita symbol $\varepsilon^{i j k}$ which is equal to $+1(-1)$ if $i, j, k$ is an even permutation (odd) the $1,2,3$, and cancels out if any two indices are equal, we can rewrite (20) such as

$\varepsilon^{i j k} \partial_{j} F_{k 0}=0$

where $F_{k 0}=-F^{k 0}=F^{0 k}=E^{k}$.

In terms of the fully antisymmetric tensor of order four $\in^{\mu v \rho \lambda}$, com $\in^{0123}=+1$, the Equation (28) it becomes $\left(\epsilon^{0 i j k}=\varepsilon^{i j k}\right)$ 
$\in^{i j k 0} \partial_{j} F_{k 0}=0$

We can now explored Einstein's relativity principle by requiring invariance of (29) under Lorentz transformation. It then follows that

$\epsilon^{\mu v \rho \lambda} \partial_{v} F_{\rho \lambda}=0$

where the covariant tensor $F_{\alpha \beta}$ it is defined as $F_{\alpha \beta}=\eta_{\alpha \mu} \eta_{\beta v} F^{\mu v}$, being $\eta_{\alpha \mu}$ covariant metric tensor of special relativity.

In terms of $\tilde{F}^{\mu v} \equiv \frac{1}{2} \epsilon^{\mu v \rho i} F_{\rho i}$, where $\tilde{F}^{\mu v}$ this is dual of $F^{\mu v}$, a Eq. (30) it becomes

$\partial_{v} \tilde{F}^{\mu \nu}=0$

The Equations (25) and (31) constitute the pair of field equations proposed by Podolsky. We have seen that the field equations proposed by Podolsky, namely

$\left[1+a^{2} \square\right] \partial_{v} F^{\mu v}=j^{\mu}$ e $\partial_{v} \tilde{F}^{\mu v}=0$

where

$$
F^{\mu v}=\left(\begin{array}{cccc}
0 & E^{1} & E^{2} & E^{3} \\
-E^{1} & 0 & B^{3} & -B^{2} \\
-E^{2} & -B^{3} & 0 & B^{1} \\
-E^{3} & B^{2} & -B^{1} & 0
\end{array}\right)
$$

We may formally obtain from the generalized Coulomb's law, in equation (15), and the superposition principle, using restricted relativity. Of course, we take into account that the electric charge on a scalar remains conserved.

In this formal deduction of the Podolsky field equations, we admit that $F^{\mu v}$ is antisymmetric, appealing to the criterion of simplicity, a common habit in physics. One can show, however, that $F^{\mu v}$ is antisymmetric, without appealing to the simplicity criterion, by using the equation of motion of a particle of mass $m$ and charge $Q$ in the electromagnetic field described by Podolsky's theory, that is,

$\frac{d P^{\mu}}{d \tau}=Q F^{\mu v} u_{v}$

where $u^{\mu}=\frac{d x^{\mu}}{d \tau}$ is 4-velocity: an operational definition of the $B^{i}$ can also be obtained from (33). To conclude these remarks we would like to draw attention to the fact that many physicists in the last century, whom have been devoted to the question of obtaining Maxwell's equations from first principles. The most recent references are the works of Kobe (1986) and (NEUENSCHWAND; TURNER, 1992).

\section{Conclusion}

We show in the course of this work that Podolsky's electrodynamics, it can be obtained from first principles, using judiciously the generalized Coulomb's law, the superposition principle and special relativity. 
Finally, we would like to point out that in recent work (GOMES, 1990; GALVÃO; PIMENTEL, 1988; BARCELOS; NATIVIDADE, 1991; BARCELOS; GALVÃO; NATIVIDADE, 1991) on Podolsky's electrodynamics has erroneously considered been the presence of a tachyon ghost. This slippage is possible in this case, because the authors of these papers started from the Lagrangean

$$
\mathcal{L}^{\prime}=-\frac{1}{2}\left[\frac{1}{2} F_{\mu v} F^{\mu v}+a^{2}\left(\partial_{\lambda} F^{\mu \lambda}\right)^{2}\right]
$$

instead of the Lagrangean proposed by Podolsky, that is,

$$
\mathcal{L}=\frac{1}{2}\left[\frac{-1}{2} F_{\mu v} F^{\mu v}+a^{2}\left(\partial_{\lambda} F^{\mu \lambda}\right)^{2}\right] .
$$

The symmetric momentum-energy tensor associated with the Podolsky Lagrangian is given by

$$
\begin{aligned}
& T_{\mu v}=\frac{a^{2}}{2} \eta_{\mu v}\left[\left(\partial_{\lambda} F^{\alpha \lambda}\right)^{2}+F_{\alpha \lambda} \square F^{\lambda \alpha}\right]-a^{2}\left[\partial_{\sigma} F_{\mu}^{\sigma} \partial_{\lambda} F_{v}^{\lambda}+F_{\mu}^{\alpha} \square F_{\alpha v}+F_{v}^{\alpha} \square F_{\alpha \mu}\right]+ \\
& +\frac{1}{4}\left(F_{\alpha \beta}\right)^{2} \eta_{\mu v}-F_{\mu}^{\alpha} F_{v}^{\alpha}
\end{aligned}
$$

A direct calculation shows that in the electrostatic case: $\vec{E}=0$ and $\vec{B}=0, T_{00}>0$; hence implying that the energy is positive definite. If we admit that the limit

$$
\vec{E} \cdot \nabla \cdot \vec{E} \rightarrow 0
$$

tends to zero faster than $\frac{1}{r^{2}}$ at infinity, we obtain the following expression for the total energy

$$
E=\int d^{3} x T^{00}=\frac{1}{2} \int d^{3} x\left[\vec{E}^{2}+a^{2}(\nabla \cdot \vec{E})^{2}\right]
$$

which in the case of a point load gives the result $\frac{Q^{2}}{2 a}$. Evidently such results are no longer true if we work with the Lagrangian $\mathcal{L}^{\prime}$.

In fact, knowing whether there is, a Lagrangian associated with a given equation of motion, and more pragmatically, knowing how to determine it if it does, is a matter of great interest to quantum field theory, where quantization methods, in their greatest part, presuppose the existence of a Lagrangian. Could it be, then, that there is some simple criterion, which allows us to decide whether a given equation of motion admits a Lagrangian? The answer is affirmative, in Accioly's article is an example (ACCIOLY et al, 1995).

\section{Acknowledgments}

The author appreciates the financial support from FAPESB/BOL 0034/2020 and CNPq.

\section{References}

Accioly, A. J., Mukai, H., Negrini, O. N., \& Sales, J. H. O. (1995). Newton's gravity and the theories with and arbitrary R coupling. Brazilian Journal of Physics, Brazil, 25(1), 76-79.

Barcelos - Neto, J., \& Braga, N. R. F. (1989). Dirac quantization of the vector superfield. Phys. Rev. D 39(2), 494. https://doi.org/10.1103/PhysRevD.39.494

Barcelos - Neto, J., \& Natividade, C. P. (1991). Schwinger model with higher derivative couplings. Z. Phys. C - Particles and Fields 49, 511-514. https://doi.org/10.1007/BF01549705 
Barcelos - Neto, J, Galvão, C. A. P., \& Natividade, C. P. (1991). Quantization of Podolsky theory in the BFV formalism. Z. Phys. C - Particles and Fields 52 (4), 529-562. https://doi.org/10.1007/BF01562328

Bartlett, D. F., \& Ward, B. F. L. (1977). Is an electron's charge independent of its velocity? Phys. Rev D 16(12), 3453. https://doi.org/10.1103/PhysRevD.16.3453

Bartlett, D. F, Shepard, J., Zafiratos, C., \& Ward, B. F. L. (1979). New limit on independence of charge and velocity. Phys. Rev. D 20(2), 578. https://doi.org/10.1103/PhysRevD.20.578

Bjorken, J. D., \& Drell, S. D. (1964). Relativistic Quantum Mechanics. McGraw-Hill Book Company, Inc.

Courant, R., \& Hilbert, D. (1962). Methods of Mathematical Physics, vol. II, Interscience Publishers, N. York and London.

Feynman, R. (1965) The Character of Physical Law. British Broadcasting Corporation 35 Marylebone High Street.

Galvão, C. A. P.; Pimentel, B. M. (1988). The canonical structure of Podolsky generalized electrodynamics. Can. J. Phys. 66, 460. https://doi.org/10.1139/p88-075

Gomes, M. O. C. (2015). Teoria Quântica de Campos, EDUSP.

Kobe, D. H. (1986). Generalization of Coulomb's law to Maxwell's equations using special relativity. Am. J. Phys. 54(7), 631. https://doi.org/10.1119/1.14521

Neuenschwander, D. E., \& Turner, B. N. (1992). Generalization of the Biot-Savart law to Maxwell's equations using special relativity. Am. J. Phys. 60(1), 35. https://doi.org/10.1119/1.17039

Podolsky, B. A Generalized Electrodynamics Part I-Non-Quantum. (1942). Phys. Rev. 62(1), 68. https://doi.org/10.1103/PhysRev.62.68

Podolsky, B., \& Kikuchi, C. (1944). A Generalized Electrodynamics Part II-Quantum. Phys. Rev. 65(7), 228. https://doi.org/10.1103/PhysRev.65.228

Podolsky, B., \& Kikuchi, C. (1945). Auxiliary Conditions and Electrostatic Interaction in Generalized Quantum Electrodynamics. Phys. Rev. 67(5), 184. https://doi.org/10.1103/PhysRev.67.184

Podolsky, B, \& Schwed, P. (1948). Review of a Generalized Electrodynamics. Rev. Mod. Phys. 20; 40(1). https://doi.org/10.1103/RevModPhys.20.40

Polyakov, A. M. (1986). Fine structure of strings. Nuclear Physics B. 268(2), 406-412. https://doi.org/10.1016/0550-3213(86)90162-8

Sales, J.H.O. (1995). Da lei de Coulomb generalizada ao eletromagnetismo com derivadas de ordem mais alta via relatividade especial. 1995. 42 f. Dissertação de Mestrado, Instituto de Física Teórica de São Paulo - UNESP, São Paulo, SP, Brasil.

Sales, J.H, \& Girotto, P. H. S. (2021). Computational Model of the Fredholm Method Applied to Podolsky Quantum Wave Function. Brazilian Journal of Development, Curitiba, 7(2), p. 20386-20399. https://doi.org/10.34117/bjdv7n2-604

Sales, J. H. de O., \& Girotto, P. H. S. (2021). Algorithms for Non-Relativistic Quantum Integral Equation. The Journal of Engineering and Exact Sciences, 7(3), 12699-01-19e. http://dx.doi.org/10.18540/jcecvl7iss3pp12699-01-19e

Slavnov, A. A. (1972). Invariant Regularization of Gauge Theories. Theor. Math. Phys. 13, 10641066. https://doi.org/10.1007/BF01035526 